\title{
A Characterization of Sturmian Morphisms *
}

\author{
Jean Berstel ${ }^{1}$ and Patrice Séébold ${ }^{2}$ \\ 1 LITP, Institut Blaise Pascal, Paris \\ 2 LAMIFA, Amiens \\ France
}

\begin{abstract}
A morphism is called Sturmian if it preserves all Sturmian (infinite) words. It is weakly Sturmian if it preserves at least one Sturmian word. We prove that a morphism is Sturmian if and only if it keeps the word $b a^{2} b a^{2} b a b a^{2} b a b$ balanced. As a consequence, weakly Sturmian morphisms are Sturmian. An application to infinite words associated to irrational numbers is given.
\end{abstract}

\section{Introduction}

A one-sided infinite word is balanced if the difference of the number of occurrences of a letter in two factors of the same length never exceeds one. It is Sturmian if it is balanced and not ultimately periodic.

Sturmian words have a long history. A clear exposition of early work by J. Bernoulli, Christoffel, and A. A. Markov is given in the book by Venkov [22]. The term "Sturmian" has been used by Hedlund and Morse in their development of symbolic dynamics $[9,10,11]$. These words are also known as Beatty sequences, cutting sequences, or characteristic sequences. There is a large literature about properties of these sequences (see for example Coven, Hedlund [6], Series [20], Fraenkel et al. [8], Stolarsky [21]). From a combinatorial point of view, they have been considered by S. Dulucq and D. Gouyou-Beauchamps [7], Rauzy [16, 17, 18], Brown [3], Ito, Yasutomi [12], Crisp et al. [5] in particular in relation with iterated morphisms, and by Séébold [19], Mignosi [13]. Sturmian words appear in ergodic theory [15], in computer graphics [2], in crystallography [1], and in pattern recognition.

A morphism is Sturmian if the image of every Sturmian word is a Sturmian word. Sturmian morphisms appear in number theory in connection with the so-called substitutions of characteristic sequences. A recent account of results in this direction is given by T. C. Brown in [4]. In this paper, we show that in order to test whether a morphism $f$ is Sturmian, it suffices to check whether the single word $f\left(b a^{2} b a^{2} b a b a^{2} b a b\right)$ is balanced. This is in fact a strengthening of a result by Mignosi, Séébold [14]. The decidability is an immediate consequence. We also get a simpler proof of a theorem by Crisp et al. [5] characterizing those irrational numbers for which the characteristic sequence is a fixed point of a (Sturmian) morphism.

\footnotetext{
* Partially supported by the PRC "Mathématiques et Informatique".
} 


\section{Definitions}

Let $A=\{a, b\}$ be a two letter alphabet. $A^{*}$ is the set of (finite) words on $A$ and $\varepsilon$ is the empty word. $A^{\omega}$ is the set of infinite words on $A$ and $A^{\infty}=A^{*} \cup A^{\omega}$.

A word $w \in A^{*}$ is primitive if it is not a power of another word, i.e. if $w=u^{p}$ for $u \in A^{*}$ and $p \in \mathbb{N}$ implies $w=u$.

For any $u \in A^{*},|u|$ denotes the length of $u$ and $|u|_{x}$ denotes the number of occurrences of the letter $x$ in the word $u$.

A morphism $h$ is a mapping from $A^{*}$ into itself such that $h(u v)=h(u) h(v)$ for all words $u, v$. A morphism is nonerasing if neither $h(a)$ nor $h(b)$ is the empty word. For any morphism $f,\|f\|$ denotes the length of $f$ which is $|f(a)|+|f(b)|$. In the sequel, all morphisms $f$ will be supposed to be distinct from the null morphism which maps all letters into the empty word (thus $\|f\| \geq 1$ ). Consider the morphism $\phi$ defined by

$$
\phi(a)=a b, \quad \phi(b)=a
$$

Setting, for $n \geq 1$,

$$
u_{n}=\phi^{n}(a), \quad v_{n}=\phi^{n}(b)
$$

it is easily seen that $u_{n+1}=u_{n} u_{n-1}, v_{n+1}=u_{n}$. The morphism $\phi$ can be extended to infinite words ; it has a unique fixed point

$$
\mathbf{F}=a b a a b a b a a b a a b a b a a b a b a \ldots=\phi(\mathbf{F})
$$

For any $w \in A^{\infty}, \operatorname{Fact}(w)$ denotes the set of finite factors of $w$. Setting, for any $u, v \in A^{*}$ such that $|u|=|v|, \delta(u, v)=\left.|| u\right|_{a}-|v|_{a} \mid$, we call balanced a word $w \in A^{\infty}$ such that $\delta(u, v) \leq 1$ for any $u, v \in F a c t(w)$ with $|u|=|v|$.

A word $\mathbf{x} \in A^{\omega}$ is Sturmian if it is a non ultimately periodic balanced word. It is a well-known property that

Property 1. The word F is Sturmian.

Sturmian words are intimately related to cutting sequences in the plane (also known as Beatty sequences). Let $\alpha, \rho$ be real numbers with $0 \leq \alpha<1$. Then the infinite word $\mathbf{f}_{\alpha, \rho}=a_{0} a_{1} \cdots a_{n} \cdots$ defined by

$$
a_{n}= \begin{cases}a & \text { if }\lfloor\alpha(n+1)+\rho\rfloor=\lfloor\alpha n+\rho\rfloor \\ b & \text { otherwise }\end{cases}
$$

is Sturmian. The special case $\rho=\alpha$ has additional properties. In this case, we write $\mathbf{s}_{\alpha}$ for $\mathbf{f}_{\alpha, \alpha}$. The word $\mathbf{s}_{\alpha}$ is the characteristic sequence of $\alpha$. Those words $\mathbf{s}_{\alpha}$ that are fixed points of morphisms have been characterized by Crisp et al. [5].

A morphism $h$ is called Sturmian if $h(\mathbf{x})$ is Sturmian for every Sturmian word $\mathbf{x}$. The morphism $I d_{A}$ and the morphism $E$ that exchanges the letters $a$ and $b$ are obviously Sturmian. Let $\tilde{\phi}$ be the morphism defined by

$$
\tilde{\phi}(a)=b a \quad \tilde{\phi}(b)=a
$$

It is well-known (see e.g. Séébold [19]) that 
Property 2. The morphisms $\phi$ and $\tilde{\phi}$ are Sturmian.

A morphism $h$ is called weakly Sturmian if there exists at least one Sturmian word $\mathbf{x} \in A^{\omega}$ such that $h(\mathbf{x})$ is Sturmian. Obviously every Sturmian morphism is weakly Sturmian. As we shall see, in fact the converse also holds.

\section{Results}

\section{Notation}

Let $m \geq 1$ and $r \geq 1$ be two integers. In the rest of this paper, the following notation will be used:

$$
\begin{aligned}
& w_{m, r}=b\left(a^{m+1} b\right)^{r+1} a^{m} b\left(a^{m+1} b\right)^{r} a^{m} b \\
& w_{m, r}^{\prime}=a b\left(a^{m} b\right)^{r+1} a^{m+1} b\left(a^{m} b\right)^{r} a^{m+1} b
\end{aligned}
$$

These words are balanced and primitive. Conversely, every Sturmian word contains as a factor a word $w_{m, r}$ or $w_{m, r}^{\prime}$ (resp. $E\left(w_{m, r}\right)$ or $\left.E\left(w_{m, r}^{\prime}\right)\right)$ for some $m, r \geq 1$.

The main result of this paper is the following theorem:

Theorem 3. Let $f$ be a morphism. For every integers $m$ and $r$ with $m, r \geq 1$, the following three conditions are equivalent:

(i) $f$ is a composition of the morphisms $E$, $\phi$ and $\tilde{\phi}$;

(ii) $f\left(w_{m, r}\right)$ is a primitive balanced word;

(iii) $f\left(w_{m, r}^{\prime}\right)$ is a primitive balanced word.

This result shows that in order to test whether a morphism is Sturmian, it suffices to check the image of $w_{m, r}$ for any arbitrary $m$ and $r$, the shortest being $w_{1,1}=b a^{2} b a^{2} b a b a^{2} b a b$. Thus, we obtain

Corollary 4. A morphism $f$ is Sturmian iff the word $f\left(b a^{2} b a^{2} b a b a^{2} b a b\right)$ is primitive and balanced. In particular, it is decidable whether a morphism is Sturmian.

Another direct consequence of this result is the following

Theorem 5. Let $f$ be a morphism. The following conditions are equivalent:

(i) $f$ is a composition of the morphisms $E$, $\phi$ and $\tilde{\phi}$;

(ii) $f$ is Sturmian;

(iii) $f$ is weakly Sturmian.

This result plays a major role in the characterization of morphisms of characteristic sequences associated to irrational numbers.

Proposition 6. Let $f$ be a morphism, and let $\alpha, \beta$ be two irrational numbers with $0<\alpha, \beta<1$ such that

$$
\mathbf{s}_{\alpha}=f\left(\mathbf{s}_{\beta}\right) \text {. }
$$

Then $f$ is a product of $E$ and $\phi$. 
Observe that there is no occurrence of the morphism $\tilde{\phi}$ in the factorization given by this proposition. This is due to the following property of the words $\mathbf{s}_{\alpha}$ :

Property 7. Let $0<\alpha<1$ be an irrational number. Then the word as $s_{\alpha}$ is lexicographically less than all its proper suffixes. Symmetrically, the word bs $\mathbf{s}_{\alpha}$ is lexicographically greater than all its proper suffixes.

From these results, one can obtain rather easily the following characterization of those irrational numbers $\alpha$ whose characteristic sequence $s_{\alpha}$ is a fixed point of a morphism (which necessarily is Sturmian). This characterization is due to Crisp et al. [5]:

Theorem 8. Let $0<\alpha<1$ be an irrational number. The word $\mathbf{s}_{\alpha}$ is a fixed point of a morphism which is not the identity iff the continued fraction development of $\alpha$ has one of the following three forms:

(i) $\left[0 ; r_{0}, \overline{r_{1}, \ldots . r_{n}}\right], \quad r_{n} \geq r_{0} \geq 1$;

(ii) $\left[0 ; 1+r_{0}, \overline{r_{1}, \ldots r_{n}}\right], \quad r_{n}=r_{0} \geq 1$;

(iii) $\left[0 ; 1, r_{0}, \overline{r_{1}, \ldots, r_{n}}\right], \quad r_{n}>r_{0} \geq 1$.

\section{Proofs}

The most involved part of the paper is the proof of theorem 3. The proof is through three lemmas. We start with a definition. A morphism $f$ is called $(m, r)$ balanced if $f\left(w_{m, r}\right)$ is balanced or $f\left(w_{m, r}^{\prime}\right)$ is balanced. By Theorem 3 , these two words are either both balanced or not. The morphism $f$ is balanced if it is $(m, r)$ balanced for some integers $m, r \geq 1$.

Lemma 9. Let $f$ be a balanced morphism. If $f(a)=a$ and $f(b) \in b A^{*} \cap A^{*} b$, then $f(b)=b$.

Lemma 10. Let $f$ be a balanced morphism. If $f(a) \in a A^{*} a$, then $f(b) \in a A^{*} \cup$ $A^{*} a$.

Lemma 11. Let $f$ and $g$ be two morphism such that $f=\phi \circ g$ or $f=\tilde{\phi} \circ g$, and let $m, r \geq 1$. Then $f$ is $(m, r)$-balanced iff $g$ is $(m, r)$-balanced.

Proof of Theorem 3: Let $f$ be a morphism. Since $E, \phi$ and $\tilde{\phi}$ are Sturmian (see e.g. Séébold [19]), it is easily seen that (i) $\Rightarrow$ (ii) and (i) $\Rightarrow$ (iii). By symmetry, it is enough to prove the implication (ii) $\Rightarrow$ (i).

Let $f$ be a morphism such that the word $f\left(w_{m, r}\right)$ is a primitive balanced word. Since $f\left(w_{m, r}\right)$ is primitive, $f(a)$ and $f(b)$ are not the empty word $\varepsilon$ thus $\|f\| \geq 2$ and the result holds for $f=I d_{A}$ and $f=E$.

Consequently, let us suppose $\|f\| \geq 3$. We observe first that $f(a)$ and $f(b)$ start or end with the same letter. Assume indeed that $f(a)$ starts with $a$ and $f(b)$ starts with $b$ (if $f(a)$ starts with $b$ and $f(b)$ starts with $a$, then consider $E \circ f)$. From Lemmas 9 and 10 it follows that if $f(a)$ and $f(b)$ do not end with the same letter, then $f(a)$ ends with $b$ and $f(b)$ ends with $a$. But in this case, 
$f(a b)$ contains the factor $b b$ and $f(b a)$ contains the factor $a a$, which contradicts the hypothesis $f\left(w_{m, r}\right)$ balanced. Consequently, let us suppose that $f(a)$ and $f(b)$ both start with the letter $a$ (if it is the letter $b$ then consider the morphism $E \circ f$ and if $f(a)$ and $f(b)$ end with the same letter, $\phi$ is replaced in what follows by $\tilde{\phi}$ ). Furthermore, let us suppose that $f(a)$ and $f(b)$ do not contain the factor $b b$. Then $f(a), f(b) \in\{a, a b\}^{*}$, thus there exist two words $x$ and $y$ such that $f(a)=\phi(x), f(b)=\phi(y)$. Denoting by $g$ the morphism defined by

$$
g(a)=x \quad g(b)=y
$$

one obtains $f=\phi \circ g$ (if $f(a)$ or $f(b)$ contains the factor $b b$ then $f=E \circ \tilde{\phi} \circ$ $g$ ). Now, Lemma 11 implies that $g\left(w_{m, r}\right)$ is balanced. Furthermore, $g\left(w_{m, r}\right)$ is primitive (since $f\left(w_{m, r}\right)$ is so) thus $|g(a b)|_{a} \neq 0$. Consequently, $\|f\|>\|g\|$ and the result follows by induction.

Proof of theorem 5: The implications (i) $\Rightarrow$ (ii) and (ii) $\Rightarrow$ (iii) are clear and we have only to prove (iii) $\Rightarrow$ (i). So let $f$ be a weakly Sturmian morphism and $\mathbf{x}$ a Sturmian word such that $f(\mathbf{x})$ is Sturmian. Furthermore, let us suppose that $\mathbf{x}$ contains the factor $a a$. In this case, there exist some integers $m$ and $r, m, r \geq 1$, such that $\mathbf{x}$ contains $w_{m, r}$ or $w_{m, r}^{\prime}$ as a factor. It follows, from theorem 3 , that $f$ can be obtained by composition of $E, \phi$ and $\tilde{\phi}$. If $\mathbf{x}$ contains the factor $b b$, then the above proof holds for $g=f \circ E$ and, consequently, $f=g \circ E$ has the required property.

Proof of Lemma 9: Let $f$ be a morphism such that $f(a)=a$ and $f(b) \in$ $b A^{*} \cap A^{*} b$, and let $m$ and $r$ be two integers, $m, r \geq 1$ such that $f\left(w_{m, r}\right)$ or $f\left(w_{m, r}^{\prime}\right)$ is a balanced word. Since $f(a)=a$, both $f\left(w_{m, r}\right)$ and $f\left(w_{m, r}^{\prime}\right)$ contain the factors $a^{m}$ and $a^{m+1}, m \geq 1$, thus $f(b)$ does not contain $b b$ and if $f(b) \neq b$ then $f(b)$ starts (resp. ends) with $b a^{m} b$ or $b a^{m+1} b$.

For all integers $p \geq 0, p^{\prime} \geq 1$, define

$$
u_{p, p^{\prime}}=b\left(a^{m+1} b\right)^{p+1}\left(a^{m} b\right)^{p^{\prime}} a^{m+1}, \quad v_{p, p^{\prime}}=b\left(a^{m} b\right)^{p^{\prime}}\left(a^{m+1} b\right)^{p} a^{m} b
$$

The word $w_{m, r}$ contains both $u_{r, 1}$ and $v_{r, 1}$, and $w_{m, r}^{\prime}$ contains both $u_{0, r}$ and $v_{0, r}$. If $f(b)$ starts with $b a^{m} b$ then $f\left(u_{p, p^{\prime}}\right)$ contains the factor

$$
z=a^{m+1} f\left(b\left(a^{m+1} b\right)^{p}\left(a^{m} b\right)^{p^{\prime}}\right) a^{m+1}
$$

and $f\left(v_{p, p^{\prime}}\right)$ contains the factor $z^{\prime}=f\left(b\left(a^{m} b\right)^{p^{\prime}}\left(a^{m+1} b\right)^{p}\right) a^{m} b a^{m} b$.

Otherwise, $f(b)=b a^{m+1} b v$ for some word $v$. But in this case $f\left(u_{p, p^{\prime}}\right)$ contains the factor $z=a^{m+1} b v f\left(\left(a^{m+1} b\right)^{p}\right) a^{m+1} b a^{m+1}$ and, since $p^{\prime} \geq 1, f\left(v_{p, p^{\prime}}\right)$ contains the factor $z^{\prime}=b a^{m} b a^{m+1} b v f\left(\left(a^{m+1} b\right)^{p}\right) a^{m} b$.

In both cases, $\delta\left(z, z^{\prime}\right)=2$ which contradicts the hypothesis that $f$ is $(m, r)$ balanced. Thus $f(b)=b$ and the lemma is proved.

Proof of Lemma 10 : Let $f$ be a morphism such that $f(a) \in a A^{*} a$ and let $m$ and $r$ be two integers, $m, r \geq 1$ such that $f\left(w_{m, r}\right)$ or $f\left(w_{m, r}^{\prime}\right)$ is a balanced word. We set, for $k \in \mathbb{N}$,

$$
u_{k}=a^{m+1} b\left(a^{m} b\right)^{k} a^{m+1}, \quad v_{k}=a b\left(a^{m} b\right)^{k} a^{m} b a
$$




$$
x_{k}=b\left(a^{m+1} b\right)^{k+1}, \quad y_{k}=b a^{m} b\left(a^{m+1} b\right)^{k} a^{m} b .
$$

By construction, $w_{m, r}$ both contains $u_{0}, v_{0}$, and $x_{r}, y_{r}$. Symmetrically, $w_{m, r}^{\prime}$ both contains $u_{r}, v_{r}$, and $x_{0}, y_{0}$.

Assume, by way of contradiction, that $f(b) \in b A^{*} \cap A^{*} b$. If $f(a)=u a a v$ then $f\left(a^{m+1}\right)$ contains the factor $z=a a v f\left(a^{m-1}\right) u a a$ and $f\left(b a^{m} b\right)$ contains the factor $z^{\prime}=$ buaav $f\left(a^{m-1}\right) b$. Then $\delta\left(z, z^{\prime}\right)=2$ which contradicts the hypothesis. Thus $f(a)$ does not contain $a a$ and, since $f(a) \neq a$, there exists an integer $n \geq 0$ such that

$$
f(a)=(a b)^{n} a b a
$$

But, in this case, $f(a b)$ contains $b a b$ and $f(a a)$ contains $b a a b$. Thus $f(b)$ does not contain $b b$ nor $a^{3}$, which implies that either $f(b)=b$ or $f(b)$ starts with $b a b$ or baab.

We shall now prove that $f(b)=(b a)^{n^{\prime}} b$, for some $n^{\prime} \geq 0$. This holds if $f(b)=b$. Thus, assume by way of contradiction that $f(b)=u a a v$ for some $u$ and $v$.

Observe first that $f(b)$ starts and ends with $b a a b$. Indeed, if $f(b)$ starts with $b a b$ then $f\left(b a^{m+1}\right)$ contains the factor $z=a a v f\left(a^{m}\right) a$ and $f\left(b a^{m} b\right)$ contains the factor $z^{\prime}=v f\left(a^{m}\right) b a b$. If $f(b)$ ends with $b a b$ then $f\left(a^{m+1} b\right)$ contains the factor $z=a f\left(a^{m}\right) u a a$ and $f\left(b a^{m} b\right)$ contains the factor $z^{\prime}=b a b f\left(a^{m}\right) u$. In both cases, $\delta\left(z, z^{\prime}\right)=2$ which contradicts the assumption.

Thus $f(b)$ starts and ends with $b a a b$. If $m=1$ then let $f(b)=b a a b v^{\prime}$ for some $v^{\prime}$. In this case, $f\left(x_{k}\right)$ contains the factor

$$
z=a^{2} b v^{\prime} f\left(\left(a^{m+1} b\right)^{k} a^{m+1}\right) b a^{2}
$$

and $f\left(y_{k}\right)$ contains the factor

$$
z^{\prime}=b f\left(a^{m}\right) b a^{2} b v^{\prime} f\left(\left(a^{m+1} b\right)^{k} a^{m}\right) b .
$$

Then $\delta\left(z, z^{\prime}\right)=2$ which contradicts the hypothesis.

Otherwise $m>1$. In this case, both $f\left(w_{m, r}\right)$ and $f\left(w_{m, r}^{\prime}\right)$ contain both of the words $f\left(a^{3}\right)$ and $f\left(a^{2} b\right)$. But $f\left(a^{3}\right)$ contains the factor $b a a(b a)^{n} b a a b$ and $f\left(a^{2} b\right)$ contains the factor $b a a(b a)^{n+1} b a a b$. Consequently, if $f(b)$ contains as a factor a power of $b a$, then this power is $(b a)^{n}$ or $(b a)^{n+1}$. Thus there exist two integers $p, p^{\prime} \geq 0$, such that $f(b)$ starts with $\left(b a a(b a)^{n}\right)^{p}\left(b a a(b a)^{n+1}\right)^{p^{\prime}} b a a b$ (remark that, if $p^{\prime}=0$ then $\left.f(b)=\left(b a a(b a)^{n}\right)^{p} b a a b\right)$.

If $p<m-1$ then $f\left(u_{k}\right)$ contains the factor

$$
z=a(a b)^{n} a b a(a b)^{n} a b a\left((a b)^{n} a b a\right)^{p} f\left(b\left(a^{m} b\right)^{k} a^{m}\right) a
$$

and $f\left(v_{k}\right)$ contains the factor

$$
z^{\prime}=b a(b a)^{n} f\left(b\left(a^{m} b\right)^{k} a^{m}\right)\left(b a a(b a)^{n}\right)^{p} b a a(b a)^{n} b a b .
$$

If $p \geq m-1$ then $f(b)$ starts with $\left(b a a(b a)^{n}\right)^{m-1} b a a$ and we denote by $v^{\prime}$ the word such that $f(b)=b v^{\prime}$. In this case, $f\left(x_{k}\right)$ contains the factor

$$
z=v^{\prime} f\left(\left(a^{m+1} b\right)^{k} a^{m}\right) f(a)\left(b a a(b a)^{n}\right)^{m-1} b a a
$$


and $f\left(y_{k}\right)$ contains the factor

$$
z^{\prime}=b f\left(a^{m}\left(b a^{m+1}\right)^{k}\right) b v^{\prime} f\left(a^{m}\right) b
$$

and $\delta\left(f\left(a^{m}\right), f(a)\left(b a a(b a)^{n}\right)^{m-1}\right)=0$. Thus, in both cases, $\delta\left(z, z^{\prime}\right)=2$ which contradicts the hypothesis.

Consequently $f(b)$ does not contain $a a$, thus $f(b)=(b a)^{n^{\prime}} b$, for some $n^{\prime} \geq 0$. But in this case, since $m \neq 0$, the word $f\left(u_{k}\right)$ contains the factor

$$
z=a f\left(\left(a^{m} b\right)^{k+1}\right)(a b)^{n} a b a a
$$

and $f\left(v_{k}\right)$ contains the factor

$$
z^{\prime}=b a b f\left(\left(a^{m} b\right)^{k+1}\right)(a b)^{n} a b .
$$

Again, $\delta\left(z, z^{\prime}\right)=2$ which contradicts the hypothesis, thus $f(b) \in a A^{*} \cup A^{*} a$ and the lemma is proved.

Proof of Lemma 11: The "only if" part is straightforward. For the "if" part, assume $f=\phi \circ g$ (the case $f=\tilde{\phi} \circ g$ could be done exactly in the same way). If $g\left(w_{m, r}\right)$ is not balanced then there exist two words $u$ and $v$ such that $g\left(w_{m, r}\right)=$ $u_{1} u u_{2}=v_{1} v v_{2}$ with $|u|=|v|$ and $\delta(u, v)=2$. Furthermore, $u$ can be choosen of minimal length, which implies that there exist $x, y \in A, x \neq y$ and $t, t^{\prime} \in A^{*}$ such that $u=x t x, v=y t^{\prime} y$ and $\delta\left(t, t^{\prime}\right)=0$. Let us assume $x=a$ and $y=b$ (the other case is exactly the same). Then $g\left(w_{m, r}\right)=u_{1} a t a u_{2}=v_{1} b t^{\prime} b v_{2}$ and $f\left(w_{m, r}\right)=\phi\left(u_{1}\right) a b \phi(t) a b \phi\left(u_{2}\right)=\phi\left(v_{1}\right) a \phi\left(t^{\prime}\right) a \phi\left(v_{2}\right)$.

If $v_{2} \neq \varepsilon$, then $\phi\left(v_{2}\right)$ starts with $a$ and $f\left(w_{m, r}\right)$ is not balanced, contradiction.

If $v_{2}=\varepsilon$, then $b t^{\prime} b$ is a suffix of $g\left(w_{m, r}\right)$. Two cases arise:

- If $\left|b t^{\prime} b\right| \leq\left|g\left(a^{m} b\left(a^{m+1} b\right)^{r} a^{m} b\right)\right|$ then $g\left(a^{m} b\left(a^{m+1} b\right)^{r} a^{m} b\right)=v_{1}^{\prime} b t^{\prime} b$ and $g\left(w_{m, r}\right)=g(b a) v_{1}^{\prime} b t^{\prime} b g\left(\left(a^{m+1} b\right)^{r} a^{m} b\right)$ which is the same case as $v_{2} \neq \varepsilon$.

- If $\left|b t^{\prime} b\right|>\left|g\left(a^{m} b\left(a^{m+1} b\right)^{r} a^{m} b\right)\right|$ then, since $m \geq 1$, one has $|a t a|>$ $\left|g\left(b\left(a^{m+1} b\right)^{r+1}\right)\right|$. Consequently, there exist three words $z, z^{\prime}, z^{\prime \prime}$ with $z \neq \varepsilon$ and $\left|z^{\prime}\right|=\left|z^{\prime \prime}\right|$ such that ata $=z^{\prime} z$ and $b t^{\prime} b=z z^{\prime \prime}$. But $\delta\left(z^{\prime}, z^{\prime \prime}\right)=\delta(u, v)=2$, and since $z \neq \varepsilon$, one has $\left|z^{\prime}\right|<|u|$ which contradicts the minimality of $|u|$.

If $g\left(w_{m, r}^{\prime}\right)$ is not balanced then the same contradiction holds when we compare $\left|b t^{\prime} b\right|$ and $\left|g\left(a^{m} b\left(a^{m} b\right)^{r} a^{m+1} b\right)\right|$, thus the lemma is proved.

We now turn to the proofs of the number-theoretic applications. Given two infinite words $\mathbf{x}=a_{0} a_{1} \cdots$ and $\mathbf{y}=b_{0} b_{1} \cdots$ over the alphabet $A=\{a, b\}$, ordered by $a<b$, we write $\mathbf{x}<\mathbf{y}$ when $\mathbf{x}$ is lexicographically less then $\mathbf{y}$, that is when there exists an integer $n$ such that $a_{n}<b_{n}$ and $a_{i}=b_{i}$ for $0 \leq i<n$. Property 7 is a consequence of the more general

Lemma 12. Let $0 \leq \rho, \rho^{\prime}<1$ and $0<\alpha<1$, with $\alpha$ irrational. Then

$$
\mathbf{f}_{\alpha, \rho}<\mathbf{f}_{\alpha, \rho^{\prime}} \Longleftrightarrow \rho<\rho^{\prime} \text {. }
$$


Proof. Since $\alpha$ is irrational, one has $\rho<\rho^{\prime}$ if and only if there exists an integer $n$ such that $\left\lfloor\alpha n+\rho^{\prime}\right\rfloor=1+\lfloor\alpha n+\rho\rfloor$ Let $m$ be the smallest integer $n$ satisfying this relation, and set $k=m-1$. Then, setting $\mathbf{f}_{\alpha, \rho}=a_{0} a_{1} \cdots$ and $\mathbf{f}_{\alpha, \rho^{\prime}}=a_{0}^{\prime} a_{1}^{\prime} \cdots$, one gets $a_{j}=a_{j}^{\prime}$ for $0 \leq j<k$ and $a_{k}<a_{k}^{\prime}$. This proves the lemma.

Proof of Property 7. Let us prove the first inequality, namely that $a \mathbf{s}_{\alpha}<\mathbf{x}$ for any proper suffix $\mathbf{x}$ of $a \mathbf{s}_{\alpha}$. For this, observe that $a \mathbf{s}_{\alpha}=\mathbf{f}_{\alpha, 0}$, and that $\mathbf{x}=\mathbf{f}_{\alpha, n \alpha-\lfloor n \alpha\rfloor}$ for some integer $n>0$. Since $\alpha$ is irrational, the conclusion follows from the preceding lemma. The other inequality is shown symmetrically.

Before proceeding to the proof of Proposition 6, recall the following relations which are well-known (see e.g. [3]) :

$$
E\left(\mathbf{s}_{\beta}\right)=\mathbf{s}_{1-\beta}, \quad \phi\left(\mathbf{s}_{\beta}\right)=\mathbf{s}_{(1-\beta) /(2-\beta)}
$$

Proof of Proposition 6. By induction on the length $\|f\|$ of $f$. We may assume that $s_{\beta}$ contains the factor $a a$. Otherwise we replace $s_{\beta}$ by $s_{1-\beta}=E\left(s_{\beta}\right)$ and $f$ by $f \circ E$. We also can suppose that $\mathbf{s}_{\alpha}$ contains the factor $a a$. Otherwise, we replace $f$ by $E \circ f$ and $\mathbf{s}_{\alpha}$ by $\mathbf{s}_{1-\alpha}$. These normalisations do not increase the length of $f$. Since $b \mathbf{s}_{\beta}$ is Sturmian, the word $\mathbf{s}_{\beta}$ starts with the letter $a$. Similarly, $\mathbf{s}_{\alpha}$ starts with the letter $a$. In particular, $f(a)$ starts with an $a$, and neither $f(a)$ nor $f(b)$ contain a factor $b b$.

If the word $f(b)$ also starts with the letter $a$, then both $f(a)$ and $f(b)$ are products of words in $\{a b, a\}$. Thus $f=\phi \circ g$ for some shorter morphism $g$, and an appropriate word $s_{\gamma}$. To conclude, it suffices to prove that $f(b)$ cannot start with a letter $b$. Indeed, otherwise $f(a)$ and $f(b)$ finish with the same letter. If this letter is a $b$, then $\mathrm{s}_{\alpha}$ contains the factor $b b$. Thus $f(a)$ and $f(b)$ finish by an $a$. Now let $r \geq 1$ by the integer such that $a^{r} b$ is a prefix of $s_{\beta}$. Then $a^{r+1} b$ is a factor of $s_{\beta}$. The word $a f\left(a^{r}\right) b$ is a prefix of $a s_{\alpha}$, and $a f\left(a^{r}\right) a$ is a factor of $\mathbf{s}_{\alpha}$. But this shows that $a \mathbf{s}_{\alpha}$ is lexicographically greater than one of its suffixes. Contradiction.

We conclude with a proof of Theorem 8. Our proof is shorter than, though not very different from [5]. It will be convenient to introduce the morphism $\gamma=\phi \circ E$. Thus $\gamma(a)=a, \gamma(b)=a b$. Clearly, a morphism is a composition of $E$ and $\phi$ iff it is a composition of $E$ and $\gamma$. The morphism $\gamma$ is used in conjonction with the morphism $\theta_{m}=\gamma^{m} \circ E$. We observe that $([3,22])$

$$
\mathbf{s}_{\beta /(1+\beta)}=\gamma\left(\mathbf{s}_{\beta}\right), \quad \mathbf{s}_{1 /(m+\beta)}=\theta_{m}\left(\mathbf{s}_{\beta}\right)
$$

Proof of Theorem 8. Let

$$
\alpha=\left[0 ; r_{1}, r_{2}, \ldots\right]
$$

be the development into continued fraction of $\alpha$. Let $f$ be such that $\mathbf{s}_{\alpha}=f\left(\mathbf{s}_{\alpha}\right)$. Then $f$ is a product of the morphisms $\gamma$ and $E$. Clearly $f \neq E$ and $f$ is not a product of $\gamma$ only. Consequently,

$$
f=\gamma^{n_{1}} E \gamma^{n_{2}} \cdots E \gamma^{n_{k}} E \gamma^{n_{k+1}}
$$


for some $k \geq 1$ and $n_{1} \geq 0, n_{2}, \ldots, n_{k} \geq 1, n_{k+1} \geq 0$. We distinguish two cases.

First case: $n_{k+1} \geq 1$. Then

$$
f=\theta_{n_{1}+1} \theta_{n_{2}} \cdots \theta_{n_{k}} \gamma^{n_{k+1}-1}
$$

Since $\mathbf{s}_{\alpha}$ is a fixed point,

$$
\left[0 ; r_{1}, r_{2}, \ldots\right]=\left[0 ; 1+n_{1}, n_{2}, \ldots, n_{k}, n_{k+1}-1+r_{1}, r_{2}, \ldots\right]
$$

whence $r_{1}=1+n_{1}, r_{2}=n_{2}, \ldots, r_{k}=n_{k}, r_{k+1}=n_{k+1}+n_{1}$ and $r_{j}=r_{j+k}$ for $j \geq 2$. Thus

$$
\alpha=\left[0 ; r_{1}, \overline{r_{2}, \ldots, r_{k+1}}\right], \quad r_{k+1} \geq r_{1}
$$

which is case $(i)$ of the theorem.

Second case: $n_{k+1}=0$. Set $f^{\prime}=E f E$. Since $\mathbf{s}_{\alpha}=f\left(\mathbf{s}_{\alpha}\right)$, one has $f^{\prime}\left(E \mathbf{s}_{\alpha}\right)=$ $E \mathbf{s}_{\alpha}$ and $f\left(\mathbf{s}_{\beta}\right)=\mathbf{s}_{\beta}$ where $\beta=1-\alpha$. Now

$$
f^{\prime}=E \gamma^{n_{1}} E \gamma^{n_{2}} \cdots E \gamma^{n_{k}} \quad \text { and } n_{k} \geq 1 .
$$

This has the same form as above, excepted when $n_{1}=0$. Again, we consider two cases:

First, assume $n_{1}=0$. Then $k \geq 3$ and $f^{\prime}=\theta_{n_{2}+1} \theta_{n_{3}} \ldots \theta_{n_{k-1}} \gamma^{n_{k-1}}$ whence, using the first case, $\beta=\left[1+n_{2}, \overline{n_{3}, \ldots, n_{k-1}, n_{2}+n_{k}}\right]$ and since $n_{2} \geq 1$, one gets for $\alpha=1-\beta$ the development

$$
\alpha=\left[0 ; 1, n_{2}, \overline{n_{3}, \ldots, n_{k-1}, n_{2}+n_{k}}\right]
$$

This is case (iii) of the theorem.

Finally, assume $n_{1}>0$. Then $f^{\prime}=\theta_{n_{0}+1} \theta_{n_{1}} \cdots \theta_{n_{k-1}} \gamma^{n_{k}-1}$ with $n_{0}=0$. Applying the first case, we get $\beta=\left[0 ; 1, \overline{n_{1}, \ldots, n_{k}}\right]$ and consequently

$$
\alpha=\left[0 ; 1+n_{1}, \overline{n_{2}, \ldots, n_{k}, n_{1}}\right]
$$

which is precisely case $(i i)$ of the statement.

\section{References}

1. E. BombIERI, J. E. TAYLor, Which distributions of matter diffract? An initial investigation, J. Phys. 47 (1986), Colloque C3, 19-28.

2. J. E. BRESENHAM, Algorithm for computer control of a digital plotter, IBM Systems J. 4 (1965), 25-30.

3. T. C. Brown, A characterization of the quadratic irrationals, Canad. Math. Bull. 34 (1991), 36-41.

4. T. C. Brown, Descriptions of the characteristic sequence of an irrational, Canad. Math. Bull. 36 (1993), 15-21.

5. D. Crisp, W. Moran, A. Pollington, P. Shiue, Substitution invariant cutting sequences, Sémin. Théorie des Nombres, Bordeaux, 1993, to appear.

6. E. Coven, G. HeduUnd, Sequences with minimal block growth, Math. Systems Theory 7 (1973), 138-153. 
7. S. Dulucq, D. Gouyou-Beauchamps, Sur les facteurs des suites de Sturm, Theoret. Comput. Sci. 71 (1990), 381-400.

8. A. S. Fraenkel, M. MushKin, U. TASSA, Determination of $\lfloor n \theta\rfloor$ by its sequence of differences, Canad. Math. Bull. 21 (1978), 441-446.

9. G.A. Heduund, Sturmian minimal sets, Amer. J. Math 66 (1944), 605-620.

10. G.A. Hedlund, M. Morse, Symbolic dynamics, Amer. J. Math 60 (1938), 815866.

11. G.A. Hedlund, M. Morse, Sturmian sequences, Amer. J. Math 61 (1940), 1-42.

12. S. ITo, S. YASUTOMI, On continued fractions, substitutions and characteristic sequences, Japan. J. Math. 16 (1990), 287-306.

13. F. Mignosi, On the number of factors of Sturmian words, Theoret. Comput. Sci. 82 (1991), 71-84.

14. F. Mignosi, P. SÉÉBold, Morphismes sturmiens et règles de Rauzy, Techn. Rep. LITP-91-74, Paris, France.

15. M. QuefFÉlec, Substitution Dynamical Systems - Spectral Analysis, Lecture Notes Math.,vol. 1294, Springer-Verlag, 1987.

16. G. RAUZY, Suites à termes dans un alphabet fini, Sémin. Théorie des Nombres (1982-1983), 25-01,25-16, Bordeaux.

17. G. RAUZY, Mots infinis en arithmétique, in: Automata on infinite words (D. Perrin ed.), Lect. Notes Comp. Sci. 192 (1985), 165-171.

18. G. RAUZY, Sequences defined by iterated morphisms, in: Workshop on Sequences (R. Capocelli ed.), Lecture Notes Comput. Sci, to appear.

19. P. SÉÉBold, Fibonacci morphisms and Sturmian words, Theoret. Comput. Sci. 88 (1991), 367-384.

20. C. SERIES, The geometry of Markoff numbers, The Mathematical Intelligencer 7 (1985), 20-29.

21. K. B. Stolarsky, Beatty sequences, continued fractions, and certain shift operators, Cand. Math. Bull. 19 (1976), 473-482.

22. B. A. Venkov, Elementary Number Theory, Wolters-Noordhoff, Groningen, 1970. 